\title{
Volume 04 Number 01 September 2020 Railway Planning Double-Double Track (Case Study of Bekasi Station km 26 + 652 - Jatinegara Station km 12 + 050
}

\author{
Ido, PRASETIO \\ Faculty of Engineering, Universitas Mercu Buana Jakarta, Indonesia \\ idoprasetyo16@gmail.com \\ Andri Irfan, RIFAI \\ Faculty of Engineering, Universitas Mercu Buana Jakarta, Indonesia \\ andrirfan@yahoo.com
}

\begin{abstract}
Increasing the number of population and the development of activities will cause a growing need for space every day, this will result in physical changes and urban land use and can cause increased intensity of population movement from Megapolitan cities such as Bekasi, Bogor and Tangerang to the Metropolitan city (DKI Jakarta). Some people have now started to move from private vehicles to use public (mass) transportation such as buses, transjakarta, and electric rail trains or commonly known as KRL Commuter Line that serve Jabodetabek routes. The impact of crossing also causes buildup at each station. Train track with a single track (single track) is considered ineffective and inefficient and vulnerable to train accidents due to human error. It is necessary to develop or develop a double track to become a double-double track Bekasi Station km $26+652$ - Jatinegara Station km $12+050$. The research location is on the railway line from Bekasi Station to Jatinegara Station. The length of the train track is around $16 \mathrm{~km}$ and has 7 stations that are traversed, 5 of which are active (stop) and 2 passive stations.
\end{abstract}

Keyword: railway, structure railway, planning

\section{INTRODUCTION}

Transportation in Jakarta provides several road and toll networks to meet access for the public, but population growth and the development of the number of motorbikes and cars with a number of roads is very lame. Congestion in Jakarta is already unavoidable due to the increasing volume of vehicles every day. Some people have now started to move from private vehicles to use public (mass) transportation such as buses, transjakarta, and electric rail trains or commonly known as KRL Commuter Line that serve Jabodetabek routes.

$\mathrm{KRL}$ is indeed a popular transportation that is often used by the community because of the short travel time compared to buses and transjakarta. Based on data from PT Kereta Commuter Indonesia (KCl) and the Central Statistics Agency (BPS, 2018) the number of $\mathrm{KRL}$ throughout 2017 reaches 315.8 million passengers every day. Based on the lane, in 2017 most KRL served passengers on the Bogor-Depok route, reaching 69.95\%, then the Bekasi-Cikarang route by $13.48 \%$, Serpong-Rangkasbitung $11.87 \%$ and Tangerang 4.72 of the total passengers.

As a result, $\mathrm{KRL}$ trains often experience delays due to having to wait for Railroad crossings to cross out or enter the station and while $K R L$ waits at the station to get a safe signal from the new Railroad Travel Regulator (PPKA), the KRL can cross, this is in accordance with the Law number 23 of 2007 concerning Railways. The impact of crossing also causes buildup at each station. Train track with a single track (single track) is considered ineffective and inefficient and vulnerable to train accidents due to human error. Need for the development or development of a double track (double track) into a doubledouble track (double-double track). (Directorate General, 2014)

(C) The Author(s). 2020 Open Access. This article is distributed under the terms of the Creative Commons Attribution 4.0 International License (http://creativecommons.org/licenses/by/4.0/), which permits unrestricted use, distribution, and reproduction in any medium, provided you give appropriate credit to the original author(s) and the source, provide a link to the Creative Commons license, and indicate if changes were made. 


\section{Volume 04 Number 01 September 2020}

Based on the above writing, this writing aims to get the geometric planning of the Bekasi Railway Station km 26 + 652 - Jatinegara Station km $12+050$ according to Service Regulation No. 10 concerning Railroad Construction Planning and Obtaining the method of implementation based on the Minister of Transportation Regulation No. 60 of 2012 and Dinas Regulation No. 10 concerning Railroad Construction Planning. As well as to add insight and knowledge in the planning and methods of implementing a good and efficient railroad and provide new reference material for civil engineering students to be used as media only.

\section{Study Literatur}

A. Railway Classification

Railway is classified based on annual transhipment capacity (Table. 1)

Table. 1 Railway Classification

\begin{tabular}{|c|c|c|c|c|c|c|c|c|}
\hline $\begin{array}{l}\text { Class } \\
\text { Street }\end{array}$ & $\begin{array}{l}\text { Traffic } \\
\text { Capacity } \\
\text { (ton } \\
\text { year) }\end{array}$ & $\begin{array}{c}\mathrm{V} \\
\text { maks } \\
(\mathrm{kmh} h \\
\text { our })\end{array}$ & $\begin{array}{c}\mathrm{p} \\
\text { Maks } \\
\text { axle } \\
\text { (ton) }\end{array}$ & $\begin{array}{l}\text { Rail } \\
\text { Type }\end{array}$ & \begin{tabular}{|c|} 
Bearing \\
Type \\
Distance \\
Between \\
Axis \\
Bearings \\
(cm) \\
\end{tabular} & $\begin{array}{l}\text { Type } \\
\text { Fastening }\end{array}$ & $\begin{array}{l}\text { Top } \\
\text { Thid: } \\
\text { Balas } \\
\text { (cm) }\end{array}$ & $\begin{array}{l}\text { Width } \\
\text { Rock } \\
\text { Balas } \\
\text { (cm) }\end{array}$ \\
\hline 1 & $>20.10^{5}$ & 120 & 18 & $\begin{array}{c}\text { R80 } \\
\text { R.5 } \\
4\end{array}$ & $\frac{\text { Concrete }}{60}$ & $\begin{array}{l}\text { Double } \\
\text { Elastic }\end{array}$ & 30 & 60 \\
\hline 2 & $\begin{array}{l}10.10^{6} \\
20.10^{6}\end{array}$ & 110 & 18 & \begin{tabular}{|c|}
$R .54$ \\
R.5 \\
0 \\
\end{tabular} & $\begin{array}{c}\begin{array}{c}\text { Concrete } \\
\text { Wood }\end{array} \\
60 \\
\end{array}$ & $\begin{array}{l}\text { Double } \\
\text { Elastic }\end{array}$ & 30 & 50 \\
\hline 3 & $\begin{array}{l}5.10^{6} \\
10.10^{6}\end{array}$ & 100 & 18 & $\begin{array}{c}R .54 \\
\text { R.5 } \\
0 / \\
\text { R.42 } \\
\end{array}$ & $\frac{\begin{array}{c}\text { ConcWood } \\
\text { /Steel }\end{array}}{60}$ & $\begin{array}{l}\text { Double } \\
\text { Elastic }\end{array}$ & 30 & 40 \\
\hline 4 & $\frac{2.5 .10^{6}-}{5.10^{6}}$ & 90 & 18 & $\begin{array}{c}\text { R.54 } \\
\text { R.5 } \\
0 / \\
\text { R.42 } \\
\end{array}$ & $\begin{array}{c}\begin{array}{c}\text { Conc/Wood } \\
\text { Steel }\end{array} \\
60\end{array}$ & $\begin{array}{l}\text { Double } \\
\text { Elastic }\end{array}$ & 25 & 40 \\
\hline 5 & $<2.5 .10^{6}$ & 80 & 18 & R.42 & $\frac{\text { Wood/Sted }}{00}$ & $\begin{array}{l}\text { Single } \\
\text { Elastic }\end{array}$ & 25 & 35 \\
\hline
\end{tabular}

Source : Minister of Transportation Regulations, 2012

\section{B. Geometric Railway}

Geometric planning of the railroad will be carried out according to the speed of the plan and the measurements of the trains that will pass by taking into account several factors, namely, safety, comfort and economy.

1. Horizontal Alignment

Table. 2 Minimum allowable radius

\begin{tabular}{|c|c|c|}
\hline $\begin{array}{l}\text { Spesd of } \\
\text { the plan } \\
\text { pmintarax } \\
\text { p }\end{array}$ & $\begin{array}{l}\text { The radius of the } \\
\text { minimum is a loop } \\
\text { without a transitional } \\
\text { curve } \\
\begin{array}{l}\text { (In) }\end{array}\end{array}$ & $\begin{array}{l}\text { The minimum radius of the } \\
\text { curved circle is permitted with } \\
\text { a transitional dinple } \\
\text { (II) }\end{array}$ \\
\hline 120 & 2370 & 780 \\
\hline 110 & 1990 & 660 \\
\hline 100 & 1650 & 550 \\
\hline 90 & 1330 & 440 \\
\hline 80 & 1050 & 350 \\
\hline 70 & 810 & 270 \\
\hline 60 & 600 & 200 \\
\hline
\end{tabular}

Source : Minister of Transportation Regulations, 2012 


$$
\begin{aligned}
& L h= \\
& 0,06\left(\frac{V^{3}}{R}\right) \ldots \ldots . . \\
& h= \\
& 5,94\left(\frac{V^{3}}{R}\right) \ldots \ldots . \\
& \text { Lh }=0,01 . h . V
\end{aligned}
$$

information :

$\mathrm{Lh}=$ The minimum length of the curve $(\mathrm{m})$

$\mathrm{h}=$ Rail High $(\mathrm{mm})$

$\mathrm{V}=$ Speed of plan $(\mathrm{km} /$ hour $)$

2. Vertical Alignment

Table. 3 Minimum allowable radius

\begin{tabular}{|c|c|}
\hline $\begin{array}{c}\text { Speed Plan } \\
\text { (km/hour) }\end{array}$ & $\begin{array}{c}\text { Mininum Vertical Alignment Radil } \\
\text { (meter) }\end{array}$ \\
\hline More $>100$ & 8.000 \\
\hline Until $\geq 100$ & 6.000 \\
\hline
\end{tabular}

Source : Minister of Transportation Regulations, 2012

$$
\begin{aligned}
& X m=\frac{R}{2} \cdot \varphi \ldots \ldots \ldots \ldots \ldots \ldots \ldots \ldots \ldots \ldots \ldots \ldots \ldots \ldots \ldots \ldots \ldots \ldots \ldots \ldots \ldots \ldots \ldots \ldots \\
& Y m= \\
& \frac{R}{8} \cdot \varphi^{2} \ldots \ldots \ldots \ldots \ldots \\
& \text { information : } \\
& X m=\text { Transitional Curved Length } \\
& Y m=\text { High } \\
& \varphi=\text { (elevasi } 2 \text { - elevasi } 1)
\end{aligned}
$$

3. Arch of Transition

$$
\begin{aligned}
& L h= \\
& 0,06\left(\frac{V^{3}}{R}\right) \\
& h= \\
& 5,94\left(\frac{V^{3}}{R}\right) \text {. } \\
& \mathrm{Lh}=0,01 . h . \mathrm{V} \\
& \text { Information : } \\
& \mathrm{Lh}=\text { The minimum length of the curve }(\mathrm{m}) \\
& \mathrm{h}=\operatorname{High}(\mathrm{mm}) \\
& \mathrm{V}=\text { Speed of plan }(\mathrm{km} / \mathrm{hour})
\end{aligned}
$$

\section{Rail}

According to the length of the train tracks can be divided into three parts :

1. The standard rail has a length of 25 meters

2. Short rails are rails that have a maximum length of 100 meters

3. Long rails are rails whose minimum lengths are listed in table 4

Table. 4 Minimum allowable radius 
Volume 04 Number 01 September 2020

\begin{tabular}{|c|c|c|c|c|}
\hline \multirow{2}{*}{ Pads Type } & \multicolumn{4}{|c|}{ Rail Type } \\
\cline { 2 - 5 } & R.42 & R.50 & R.54 & R60 \\
\hline Wood Pads & $325 \mathrm{~m}$ & $375 \mathrm{~m}$ & $400 \mathrm{~m}$ & $450 \mathrm{~m}$ \\
\hline $\begin{array}{c}\text { Concrete } \\
\text { Pads }\end{array}$ & $200 \mathrm{~m}$ & $225 \mathrm{~m}$ & $250 \mathrm{~m}$ & $275 \mathrm{~m}$ \\
\hline
\end{tabular}

Source : Minister of Transportation Regulations, 2012

Table. 5 Section Rail

\begin{tabular}{|c|c|c|c|c|c|c|c|c|}
\hline $\begin{array}{c}\text { Sped } \\
\text { of } \\
\text { Plan }\end{array}$ & $\begin{array}{c}\text { V Mals } \\
(\mathrm{Gm} / \mathrm{hor})\end{array}$ & $\begin{array}{c}\mathrm{D} 1 \\
(\mathrm{cr})\end{array}$ & $\begin{array}{c}\mathrm{B} \\
(\mathrm{cr})\end{array}$ & $\begin{array}{c}\mathrm{C} \\
(\mathrm{cm})\end{array}$ & $\begin{array}{c}\mathrm{K} 2 \\
(\mathrm{~cm})\end{array}$ & $\begin{array}{c}\mathrm{D} 2 \\
(\mathrm{cr})\end{array}$ & $\begin{array}{c}\mathrm{E} \\
(\mathrm{cr})\end{array}$ & $\begin{array}{c}\mathrm{K} 2 \\
(\mathrm{cr})\end{array}$ \\
\hline I & 120 & 30 & 150 & 235 & 240 & $15-50$ & 25 & 375 \\
\hline II & 110 & 30 & 150 & 235 & 240 & $15-50$ & 25 & 375 \\
\hline III & 100 & 30 & 140 & 225 & 240 & $15-50$ & 22 & 325 \\
\hline IV & 90 & 25 & 140 & 215 & 240 & $15-35$ & 20 & 300 \\
\hline V & 80 & 25 & 135 & 210 & 240 & $15-35$ & 20 & 300 \\
\hline
\end{tabular}

4. Sleepers Rail

Table 6 Permission Required Conditions

\begin{tabular}{|l|c|c|}
\hline $\begin{array}{l}\text { Concrats } \\
\text { Quality }\end{array}$ & $\begin{array}{c}\text { Pernission Voltage Press } \\
\left(\mathrm{kg}^{\mathrm{cm}}\right)^{2}\end{array}$ & $\begin{array}{c}\text { Permission Voltage Pall } \\
\left(\mathrm{kg} / \mathrm{cm}^{2}\right)\end{array}$ \\
\hline $\mathrm{K}-350$ & 120 & 175 \\
\hline $\mathrm{K}-500$ & 200 & 35 \\
\hline
\end{tabular}

Source : Minister of Transportation Regulations, 2012

4.1 Sleeper Analysis

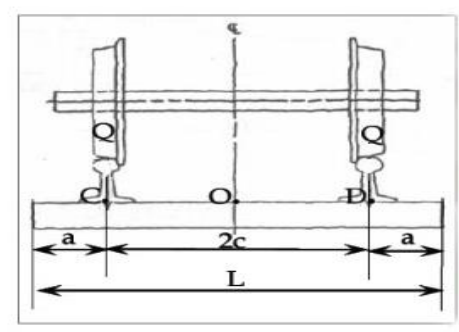

$$
\begin{aligned}
& \lambda=\sqrt[4]{\frac{k}{4 E I}} \\
& E=6400 \sqrt{F_{C U}}
\end{aligned}
$$

Picture 1 Cross section concrete pads

Information :

$$
\begin{array}{ll}
\lambda & : \text { dumping factor }\left(\mathrm{cm}^{1}\right) \\
\mathrm{E} & : \text { sleeper elastic }\left(\mathrm{kg} / \mathrm{cm}^{2}\right) \\
\mathrm{f}_{\mathrm{cu}} & : \text { concrete quality }\left(\mathrm{kg} / \mathrm{cm}^{2}\right)
\end{array}
$$




\section{Volume 04 Number 01 September 2020}

Ix :moment of inertia of the rail $x-x\left(\mathrm{~cm}^{2}\right)$

A : distance of rail center to sleeper center $(\mathrm{cm})$

C : distance of rail center to sleeper center $(\mathrm{cm})$

4. Ballas

$\mathrm{x}=\frac{\mathrm{b}}{2 \times \mathrm{h}} \times \tan \alpha \times \mathrm{P}$

Information : $\mathrm{x}=$ Voltage happens $\left(\mathrm{kg} / \mathrm{cm}^{2}\right)$

$$
\begin{aligned}
& \mathrm{b}=\text { sleeper width }(\mathrm{cm}) \\
& \mathrm{h}=\text { Thickness of Ballas }(\mathrm{cm}) \\
& \left.\alpha=\text { Angle of pressure spread (broken stone }=60^{\circ}\right) \\
& \mathrm{P}=\text { Pressure double axle }(\mathrm{kg})
\end{aligned}
$$

Terzaghi's formula for finding the carrying capacity of the soil.

qult $=1,3 . \mathrm{c} . \mathrm{Nc}+\mathrm{q} \cdot \mathrm{Nq}+0,4 \cdot \gamma \cdot \mathrm{B} \cdot \mathrm{N} \gamma$

xijin $=\frac{\text { qult }}{\mathrm{FK}}$

Information : qult = Ultimate soil carrying capacity $\left(\mathrm{kg} / \mathrm{cm}^{2}\right)$

5. Sub Ballas

$\mathrm{FK}=$ Safety factor $(\mathrm{FK}=2)$

Xijin $=$ Voltage Soil $\left(\mathrm{kg} / \mathrm{cm}^{2}\right)$

Table 7 Thickness Sub-Ballas

\begin{tabular}{|c|c|c|}
\hline $\begin{array}{c}\text { Speed } \\
(\mathrm{km} / \mathrm{hour})\end{array}$ & $\begin{array}{c}\text { Transport } \\
\text { Traffic }\end{array}$ & $\begin{array}{c}\text { Thiclcness Sub } \\
\text { Ballas } \\
\mathrm{cm}\end{array}$ \\
\hline $120-160$ & $>12$ billion & 38 \\
\hline $120-160$ & $2-12$ billion & 30 \\
\hline $120-160$ & $<2$ billion & 23 \\
\hline $80-120$ & $>12$ billion & 30 \\
\hline $80-120$ & $<12$ billion & 23 \\
\hline$<80$ & $>2$ billion & 23 \\
\hline
\end{tabular}

6. Wesel

(Source : Railway management \& engineering)

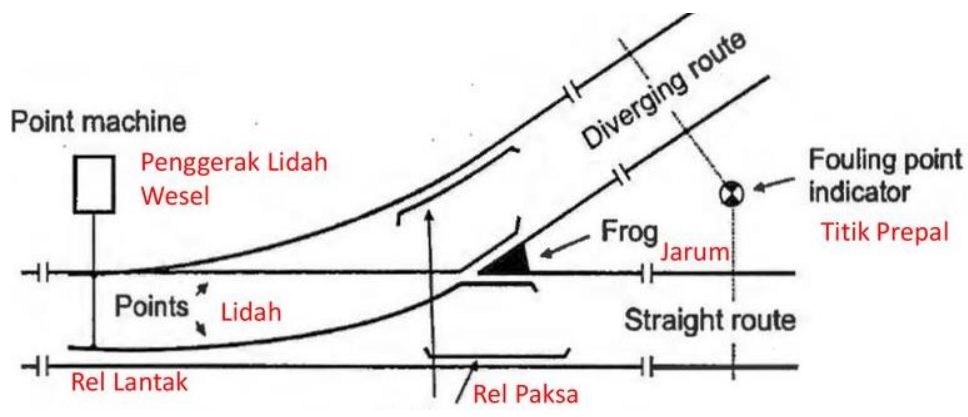

Picture 2 wesel component 
6. Rail Connection

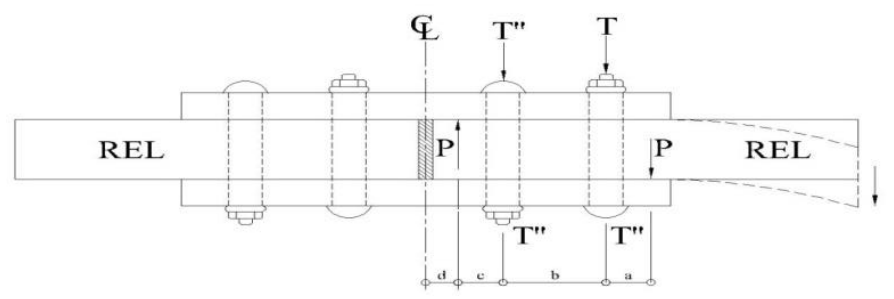

Picture 3 Rail Connection

The connecting plate bolt must withstand the following:

$$
\begin{aligned}
& H=T^{\prime}+T^{\prime \prime} \\
& M=H \times(a+b+c) \\
& M^{\prime}=H \times(a+b) \\
& M^{\prime \prime}=T^{\prime \prime} \times(a+b)+T^{\prime \prime} \times c
\end{aligned}
$$

Information:

$\mathrm{H}=$ Lateral force that works in the middle of the connection plate

T' \& T" = Tensile strength of outer and inner bolts

M' \& M" = Momentary in and out of plate between rail pressure centers

$\mathrm{M} \quad=$ Moment total lateral direction

\section{METHODOLOGY}

Field research methodology can be illustrated through the flow chart as follows:

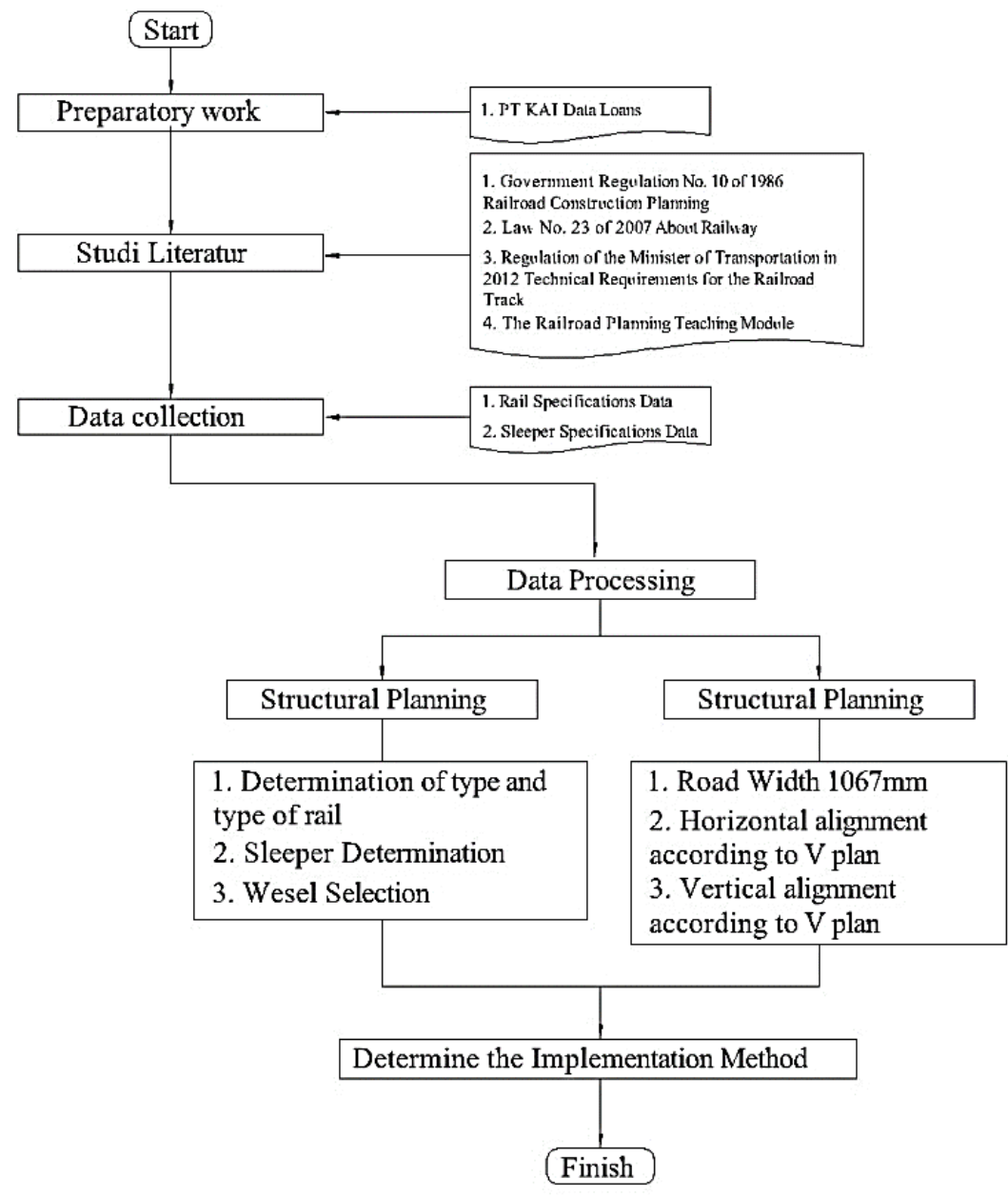




\section{Volume 04 Number 01 September 2020}

The planning data used in this Final Project is based on data obtained from related parties, in this case the relevant party is PT Kereta Api Indonesia (PT KAI) Daop 1 Jakarta. Literature studies used include :

1. Government Regulation no 10 of 1986 Railway Construction Planning

2. Law No. 23 of 2007 concerning Railways

3. Minister of Transportation Regulation No. 60 of 2012 - Technical Requirements for Railway Tracks.

4. The Railroad Planning Teaching Module

1. Calculated Geometric Planning:
A. Vertical Alignment
B. Horizontal Alignment

2. Structural planning to be carried out in the field :
A. Rail Profile Planning
Determination of rail dimensions that will be used in planning
B. Sleeper
Planning about determining the type of bearing to be used,
C. Sub Ballas and Ballas
Planning about the ballas material to be used
D. Wesel
Proper money order planning that will be used in accordance with field data.

\section{RESULTS AND DISCUSSION}

1. Horizontal Alignment Planning

In planning horizontal curvature using the spiral - circle - spiral curvilinear parameters. Examples of calculation of horizontal arcs on PV-15 Km $23+800$ to $\mathrm{Km} 23+525$, as for the steps in completing the calculation as follows.

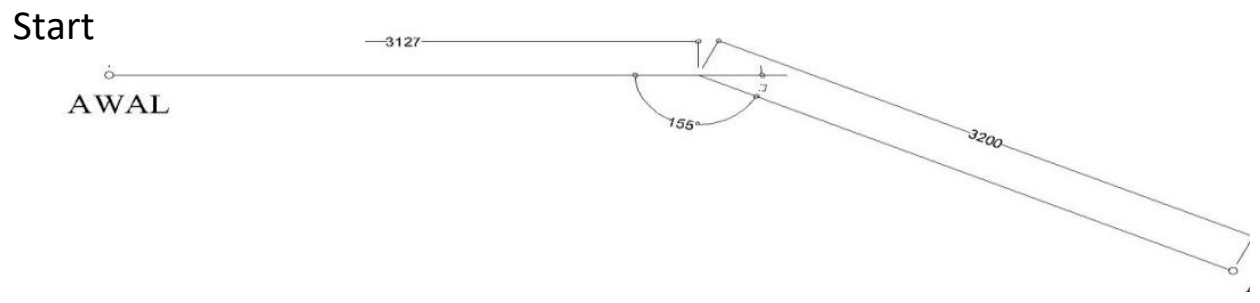

Finish

Picture 10 Horizontal Alignment

$$
\begin{array}{ll}
\text { Vplanning } & =60 \mathrm{~km} / \mathrm{jam} \\
\Delta & =180^{\circ}-155^{\circ}=25^{\circ} \\
\text { Rplanning } & =869 \mathrm{~m}
\end{array}
$$

A. Elevation of the outer side of the rail

$$
\begin{aligned}
& \mathrm{h}=5,95\left(\frac{\mathrm{V}^{3}}{\mathrm{R}}\right) \\
& \mathrm{h}=5.95\left(\frac{60^{3}}{869}\right)=24.65 \mathrm{~mm}<60 \mathrm{~mm}
\end{aligned}
$$


Volume 04 Number 01 September 2020

$B$. The minimum length of the transition arc

$$
\begin{aligned}
& \mathrm{Lh}=0,01 \times \mathrm{h} \times \mathrm{V} \\
& \mathrm{Lh}=0,01 \times 24,65 \times 60=14,79 \mathrm{~mm}
\end{aligned}
$$

C. Arc spiral

$$
\begin{aligned}
& \theta \mathrm{s}=\frac{90^{\circ} \times \mathrm{Lh}}{(\pi \times \mathrm{R})}=\frac{90 \times 14,79}{(3,14 \times 869)}=0,478^{\circ} \\
& \Delta \mathrm{c}=\Delta-2 \times \theta \mathrm{s} \\
& \Delta \mathrm{c}=25^{\circ}-\left(2 \times 0,478^{\circ}\right)=24,044^{\circ}
\end{aligned}
$$

D. Long arc circle

$$
\mathrm{Lc}=\frac{(\Delta-2 \times \theta s) \mathrm{x}(\pi \mathrm{xR})}{180}=\frac{12^{\circ}-\left(2 \times 0,478^{\circ}\right) \times(3.14 \times 869)}{180}=167,12 \mathrm{~m}
$$

E. Long transitional coordinate point length

$$
\begin{aligned}
\mathrm{p} & =\frac{\left(\mathrm{lh}^{2}\right)}{(6 \times \mathrm{R})}-\mathrm{R} \times(1 \cos \theta \mathrm{s}) \\
& =\frac{\left(14,79^{2}\right)}{(6 \times 869)}-869 \times\left(1 \cos 0,478^{\circ}\right)=0,0104 \mathrm{~m} \\
\mathrm{k} & =\mathrm{lh}-\frac{\left(\mathrm{lh}^{3}\right)}{\left(40 \times \mathrm{R}^{2}\right)}-\mathrm{R} \times(1 \sin \theta \mathrm{s}) \\
& =14,79-\frac{\left(14,79^{3}\right)}{\left(40 \times 869^{2}\right)}-869 \times\left(1 \sin 0,478^{\circ}\right)=7,390 \mathrm{~m}
\end{aligned}
$$

$F$. The distance of the starting point starts into the curved region

$$
\begin{aligned}
& \mathrm{Ts}=(\mathrm{R}+\mathrm{p}) \times \tan \left(\frac{1}{2} \times \Delta\right)+\mathrm{k} \\
& \mathrm{Ts}=(869+0,0104) \times \tan \left(\frac{1}{2} \times 25\right)+7,390=68,158 \mathrm{~m} \\
& \mathrm{E}=\frac{(\mathrm{R}+\mathrm{p})}{\cos \left(\frac{1}{2} \times \Delta\right)}-\mathrm{R}=\frac{(869+0,0104)}{\cos \left(\frac{1}{2} \times 25\right)}-869=2,132 \mathrm{~m} \\
& \mathrm{Xs}=\operatorname{Lh} \times\left(1-\frac{\mathrm{lh}^{2}}{40 \times \mathrm{R}^{2}}\right) \\
& =14,79 \times\left(1-\frac{14,79^{2}}{40 \times 869^{2}}\right)=14,789 \mathrm{~m} \\
& \text { Ys }=\frac{\mathrm{lh}^{2}}{6 \times \mathrm{R}}=\frac{14,79^{2}}{6 \times 869}=0,042 \mathrm{~m}
\end{aligned}
$$




\section{Volume 04 Number 01 September 2020}

Picture 11 Horizontal Alignment

\section{Vertical Alignment Planning}

Vertical curve is a circular element that connects two different slopes which are determined by the elevation of the height and the magnitude of the vertical arch radius

Examples of calculation of vertical arcs at Km $26+262$, as for the steps in the completion of the calculation as follows.

$$
\mathrm{e} 1=0,000
$$

Picutre 12 Vertical Alignment

$$
\begin{array}{ll}
\text { Vplanning } & =80 \mathrm{~km} / \mathrm{jam} \\
\text { Ralign } & =6000 \mathrm{~m}
\end{array}
$$

Long transitional arc $(\mathrm{Xm})$ \& high $(\mathrm{Ym})$

$$
\begin{aligned}
\mathrm{Xm} & =\frac{(\mathrm{R})}{2} \times \varphi \\
& =\frac{(6000)}{2} \times(\mathrm{e} 2-\mathrm{e} 1) \\
& =\frac{(6000)}{2} \times(-0,0233-0,000)=69,90 \mathrm{~m} \\
\mathrm{Ym} & =\frac{\mathrm{R}}{8} \times \varphi^{2} \\
& =\frac{6000}{8} \times(-0,0233-0,000)^{2}=0,4071 \mathrm{~m}
\end{aligned}
$$

\section{CONCLUSIONS}

In accordance with the analysis of data and conditions in the field of the railway track in the Planning of Double-Double Track (Jatinegara Station $(12+050)$ to Bekasi Station $(26+652)$

1. Horizontal and vertical curvilinear planning for plan speed, arch angle and radius (R) follows the data obtained from PT Kereta Api Indonesia (KAI).

2. Results of railroad structure planning
a. Road type
= Type I
b. Rail type
$=\mathrm{R} .54$
c. Vmax
$=80 \mathrm{~km} / \mathrm{hr}$
d. Axle load
$=5,6$ ton
e. Width
$=1067 \mathrm{~mm}$
f. Space of Sleeper
$=60 \mathrm{~cm}$ 


\section{Volume 04 Number 01 September 2020}

3. Method of Implementing Railway Construction:

1. Temporary Facility and Self Protective Equipment

2. Clearance Top Soil

3. Cut \& Fill Work

4. Soil Compacted

5. Work Sub-Ballas

6. Work Ballas

7. Installation Sleeper

8. Installation Rail

9. Installation Fastening

10. Work Joint Rail

\section{References}

[1] Ditjen, P. (2014). Railway Information Book. Jakarta: Djka

[2] Esveld, C. (1986). Modern Railway Track. MRT Germany.

[3] PJKA, P. (1986). Government Regulation no 10 of 1986 Railway Construction Planning

[4] Rosyidi. (2015). Universitas Muhammadiyah Yogyakarta. Rekayasa Jalan Kereta Api.

[5] Salim, A. (2012). Manajemen Transportasi. Faktor Keterlambatan Kedatangan Kereta Api Penumpang dan Kinerja Daerah Jakarta.

[6] Selig, E. \&. (1994).Track Geotechnology and Substructure Management. Thomas Telford.

[7] Undang-Undang. (2017). No. 23 Tentang Perkeretaapian. Jakarta.

[8] Wasanta, T. (2015). Desain Jalan Rel Untuk Transportasi Batu Bara Rangkaian Panjang (Studi kasus : Sumatera Selatan). 